\title{
Cooperativismo y economía del bien común
}

\author{
Alejandro Martínez Charterina \\ Catedrático de la Universidad de Deusto \\ Director del Instituto de Estudios Cooperativos de la Facultad de Derecho
}

Sumario: I. De la cooperativa singular a la presencia y protagonismo social del cooperativismo: La cooperativa moderna, el movimiento cooperativo, y las aspiraciones de conquista del cooperativismo. II. El programa de Charles Gide: la república cooperativa. III. El cooperativismo en la política. IV. El modelo de la economía del bien común y la cooperativa. V. Conclusión. VI. Bibliografía.

Resumen: En los últimos años (desde 2010) se está promocionando el modelo de economía del bien común que muestra un buen número de cooperativas como ejemplo de lo que promociona en el orden empresarial. Este trabajo trata de destacar el interés de las cooperativas por la mejora social, en general, y en particular en el medio en el que se hacen presentes, trascendiendo los intereses de cada cooperativa singular. Esto queda bien unido a las pretensiones de la Alianza Cooperativa Internacional dirigidas a la promoción de conductas que puedan llevar a un cambio social y una economía sostenible, que reconocemos como las aspiraciones de conquista del cooperativismo.

Palabras clave: cooperativismo, economía social, modelo económico del bien común.

Abstract: In recent years (since 2010), the economic model of the common good is being promoted, which shows a number of cooperatives as an example of what it promotes at a corporate level. This paper seeks to highlight the interest of cooperatives for social improvement, in general, and the context in which they are present, in particular, going beyond the interests of each individual cooperative. This is closely related to the aims of the International Cooperative Alliance to promote behaviours that can lead to social change and to a sustainable economy, which we recognise as the ultimate aims of cooperativism.

Key words: cooperativism, social economy, economic model of the common good. 


\section{De la cooperativa singular a la presencia y protagonismo social del cooperativismo: La cooperativa moderna, el movimiento cooperativo, y las aspiraciones de conquista del cooperativismo}

Es bien conocido que la doctrina considera a la cooperativa de Rochdale, The Rochdale Society of Equitables Pioneers, constituida en 1844, como el punto de partida del cooperativismo moderno, toda vez que «desde su iniciación estableció un programa completo que contenía los principios teóricos y las reglas prácticas de organización y funcionamiento de las cooperativas de consumo» ${ }^{1}$.

Aunque se conocen muchas cooperativas anteriores en el tiempo ${ }^{2}$, se concede la primacía a la de Rochdale por esa aportación que fue extendiendo los principios cooperativos a través de las nuevas cooperativas que los recogían en sus Estatutos.

Todas las cooperativas tienen un valor singular en su origen, en la medida en que conforman una asociación de personas y una empresa económica conjunta e inseparable. Personas que comparten una necesidad o aspiración y que quieren satisfacerla o alcanzarla precisamente a través de la empresa cooperativa ${ }^{3}$.

Ahora bien junto a esta dimensión singular de cada cooperativa encontramos pronto en el tiempo desde este origen del cooperativismo moderno una dimensión mayor, toda vez que las cooperativas van a tratar de unirse unas con otras para formar el movimiento cooperativo.

No se puede dejar de considerar que en los primitivos Estatutos de la Cooperativa de Rochdale se contemplan los objetivos y planes de la Sociedad que van mucho más lejos de la venta de los artículos necesarios para la vida corriente en un almacén, que es lo que realmente ponen en funcionamiento, proponiéndose la construcción de viviendas, la fabricación de bienes para dar trabajo a los socios desempleados, el cultivo de tierras con el mismo fin, y ... «Tan pronto como sea posible, esta sociedad procederá a organizar las fuerzas de la producción, distri-

1 Gromoslav MLADENATZ, Historia de las doctrinas cooperativas (1969), p. 65.

2 Puede verse una referencia a las mismas en Alejandro MARTINEZ CHARTERINA, «Evolución del cooperativismo de consumo», en Boletín de la Asociación Internacional de Derecho Cooperativo (2011), pp. 133-134.

3 En este sentido el concepto de cooperativa de la Alianza Cooperativa Internacional, en ACl, Declaración de la Alianza Cooperativa Internacional sobre la identidad cooperativa (1996), p. 17. Sobre la empresa cooperativa puede verse Alejandro MARTíNEZ CHARTERINA, «La cooperativa como empresa y los principios cooperativos tras el congreso centenario de la A.C.I. de Manchester», en Evolución del escenario económico (1996), pp. 207 ss. 
bución, educación y gobierno...», ello a través de una colonia autosuficiente. Del mismo modo se prestará ayuda a otras cooperativas con el mismo fin ${ }^{4}$.

En Gran Bretaña pocos años después de la creación de Rochdale, en 1851, se reunieron cuarenta y cuatro cooperativas en la Conferencia Nacional, en 1863 se asociaron cuarenta y ocho cooperativas en la Sociedad Cooperativa Mayorista del Norte de Inglaterra, en 1867 se creó la Sociedad Cooperativa Mayorista Escocesa, que se uniría con la primera formando la Sociedad Cooperativa Mayorista Unida Inglesa y Escocesa. A partir del Congreso Nacional Cooperativo de Londres de 1869 los congresos se celebrarían anualmente y una Junta Central Cooperativa mantendría los contactos entre congresos hasta convertirse en la Unión Cooperativa de Gran Bretaña ${ }^{5}$.

En Francia en 1884 se constituyó una organización central de las cooperativas obreras de producción, origen de la Confederación General6, y el año siguiente las cooperativas de consumo pusieron en marcha la Unión Cooperativa tras su congreso nacional ${ }^{7}$.

En Italia se constituyó la Federación Nacional de las Cooperativas en 1886, la cual se transformaría en Liga en $1893^{\circ}$. En Alemania se fundó la Unión Nacional Allgemeiner Verband, en Suiza la Unión de Consumidores Suizos para agrupar a las cooperativas de consumo, en $1890^{9}$.

De esta forma desde los primeros tiempos del cooperativismo moderno se van formando las estructuras cooperativas nacionales buscando ventajas, la unión se convierte en fuerza, tanto en el orden económico a través de centrales mayoristas para las compras en común, como en el orden político o representativo, de especial importancia en un tiempo en el que pocas personas confiaban en las cooperativas ${ }^{10}$.

Con los movimientos cooperativos nacionales en marcha no resulta extraño que se intentara su prolongación a nivel internacional, impulsada por los líderes cooperativistas que los estaban animando. Desde que en el Congreso de la cooperación inglesa, celebrado en Londres en

${ }^{4}$ Laws and objets of the Rochdale Society of Equitable Pioneers: enrolled according to the Acts, $10^{\text {th }}$, George IV, and $4^{\text {th }}$ and $5^{\text {th }}$, William IV (1884), p.3.

5 Véase en Margaret DIGBY, El movimiento cooperativo mundial (1965), pp. 31 ss.

6 Puede verse en W.P. WATKINS, L'Alliance Cooperative International 1895-1970 (1971), p. 20.

7 W.P. WATKINS, El movimiento cooperativo internacional (1977), p. 36.

8 Giulio SAPELLI, I/ movimiento cooperativo in Italia: Storia e problemi (1981), p. 27.

9 W.P. WATKINS, El movimiento... (1977), p. 36.

10 Lo que Alex Laidlaw llamó crisis de credibilidad que tuvieron que afrontar las cooperativas sobre todo en los primeros tiempos. A.F. LAIDLAW, "Las cooperativas en el año $2000 »(1982)$, p. 16. 
1869, participaron dieciocho delegados extranjeros ${ }^{11}$, se desarrollaron corrientes de relaciones personales internacionales encaminadas a la formación de un organismo internacional.

Aunque el proceso fue lento y no estuvo exento de dificultades, los intercambios a través de los Congresos nacionales alimentaron el debate en torno a la cooperación internacional y el Congreso de Londres de 1895 decidió la constitución de la Alianza Cooperativa Internacional aprobando la resolución presentada por Georges J. Holyoake, que decía: "Las organizaciones y las personas físicas que han prestado su adhesión, se comprometen por este mismo hecho, constituida ya la Alianza, a proseguir la obra comenzada por el difunto Wasittart-Neale y sus amigos» ${ }^{12}$.

Así conformado, el movimiento cooperativo no constituye únicamente una referencia acerca de la expansión y la importancia de las cooperativas, así como un instrumento capaz de fortalecerlas política y económicamente, sino que se identifica con el cooperativismo mismo, una doctrina para difundir y consolidar a las cooperativas «como forma ideal de organización de las actividades socioeconómicas de la humanidad» ${ }^{13}$.

De este modo se va desbordando la singularidad de la cooperativa que acentúa su carácter social ${ }^{14}$, nacional e internacional.

La Alianza Cooperativa Internacional es una organización no gubernamental que se caracteriza por su larga historia y su estabilidad: «... establecida en el siglo XIX ... ha resistido el paso del tiempo y todos los acontecimientos, guerras, revoluciones y cambios profundos que se han dado en el mundo ... y que ha permanecido fiel a su carácter original y a sus objetivos» ${ }^{15}$.

Los objetivos de esta organización, representante de las cooperativas a nivel mundial se recogen en el artículo 2 de su Estatuto ${ }^{16}$ :

11 Véase en Gromoslav MLADENATZ, o.c. (1969), p. 124.

12 W.P. WATKINS, L'Alliance... (1971), p. 39. Holyoake se refería en el texto de la resolución a Edward Wasittart-Neale, que había fallecido en 1892, y a Emile de Boyve, Charles Gide, y Edward Owen Greening, considerados los principales impulsores del debate que condujo a la constitución de la Alianza Cooperativa Internacional (Gromoslav MLADENATZ, o.c., p. 124.

13 Alicia KAPLAN DE DRIMER y Bernardo DRIMER, Las cooperativas: fundamentos, historia, doctrina (1981), p. 39.

14 Este carácter social que ya se contempla en el séptimo principio cooperativo de interés por la comunidad. ACl, Declaración... (1996), p. 19.

15 De este modo presentaba a la Alianza Georges DAVIDOVIC, en su obra Hacia un mundo cooperativo (1976), p. 37.

16 Aprobado por la Asamblea General en 2008 y revisado por el mismo órgano en 2009. A.C.I. Estatuto (2009), p. 1. 
«a. promover el movimiento cooperativo mundial, basado en la auto-ayuda mutua y la democracia;

b. promover y proteger los valores y principios cooperativos;

c. facilitar el desarrollo de relaciones económicas y otros beneficios mutuos entre sus organizaciones miembro;

d. promover el desarrollo humano sostenible y promover el progreso económico y social de las personas, contribuyendo así a la paz y la seguridad internacionales.

e. promover la igualdad entre hombres y mujeres en todas las tomas de decisión y actividades en el seno del movimiento cooperativo.»

Se desprenden de estas finalidades de la Alianza Cooperativa Internacional las ideas de universalidad y unidad del movimiento cooperativo, la salvaguarda de los principios y valores del cooperativismo, y la existencia de unas aspiraciones de influencia en la sociedad, que en ocasiones se han identificado como aspiraciones de conquista.

Con esta expresión "aspiración de conquista» Paul Lambert cierra las notas que ayudan a conformar la esencia de la cooperación. Lo expresa en los siguientes términos: "...queriendo, con sus métodos propios, servir a sus miembros y a la colectividad entera, la cooperación tiende a conquistar y transformar la organización económica y social del mundo. Así lo proclama la $\mathrm{ACl}$ con toda justicia» ${ }^{17}$.

El hecho de que las cooperativas, empresas mediante las cuales sus socios pretenden alcanzar unas aspiraciones o satisfacer necesidades, como se ha dicho anteriormente, conformen su conducta a unos principios cooperativos poniendo de relieve la presencia de unos valores humanos ligados a los principios, revela la existencia de una concepción del ser humano y la sociedad, y la Alianza Cooperativa Internacional, mirando hacia fuera de las cooperativas quiere hacerla patente ${ }^{18}$.

En este sentido estudia Laura Gómez las distintas preocupaciones y acciones de la Alianza que conforman las aspiraciones de conquista del cooperativismo: acciones en favor del desarrollo, actividad educativa, evolución y abordaje de los objetivos sociales desde la satisfacción de las necesidades básicas, la vivienda, la protección del consumidor, y el turismo social, hasta el medio ambiente vinculado a la sostenibilidad, la política desplegada en favor de la mujer, la especial atención por la

17 Paul LAMBERT, La doctrina cooperativa (1970), p. 272.

18 Véase Alejandro MARTíNEZ CHARTERINA, en el Prólogo de la obra de Laura GÓMEZ, La Alianza Cooperativa Internacional y su desarrollo como institución y en especial como instrumento transformador de la sociedad (1998), p. 9. 
juventud y la infancia, y la actividad en favor de la paz ${ }^{19}$. Nada de ello debe ser ajeno a los intereses globales de las cooperativas.

\section{El programa de Charles Gide: la república cooperativa}

Charles Gide, que con Emile de Boyve, formó el lado francés impulsor de la creación de la Alianza Cooperativa Internacional, fue el miembro más cualificado de la Escuela de Nimes, que había sido fundada por el propio de Boyve y por Auguste Fabre.

Se le encargó el discurso inaugural del IV Congreso del cooperativismo francés celebrado en Lyon en 1889 y en el mismo propuso un programa común que permitiera unir al centenar de cooperativas de consumo que conformaban la Unión, desviándose de las doctrinas económicas existentes entonces, es decir, la doctrina de los clásicos y la doctrina marxista.

Ese programa contenía tres etapas sucesivas:

«1) Agrupar a las sociedades ${ }^{20}$ entre sí, descontar de sus beneficios la parte mayor que se pueda para crear grandes almacenes al por mayor y realizar las compras en gran escala; he aquí la primera etapa.

2) Con los capitales que se hayan constituido así, comenzar a producir directamente todo lo necesario para cubrir las necesidades de los socios, creando panaderías, molinos, fábricas de telas y vestidos confeccionados, fábricas de zapatos, de sombreros, de jabón, de galletas, de papel...; he aquí la segunda etapa.

3) Por último, en un futuro más o menos lejano, adquirir tierras y granjas para producir directamente en ellas el trigo, el vino, el aceite, carne, leche, aves, huevos, legumbres, frutas, flores y la madera, que constituyen la base del consumo; he aquí la última etapa.

O dicho en pocas palabras, en una primera etapa victoriosa, conquistar la industria comercial; en una segunda, la industria manufacturera; por último, en una tercera, la industria agrícola; este debe ser el programa de la cooperación» ${ }^{21}$.

Gide establece como motor de este nuevo programa a las cooperativas de consumidores, lo que elimina cualquier preferencia de clase y se vincula con todas las personas, puesto que todos somos consumidores. Y, a través del mismo, se conseguiría el alejamiento de las lu-

19 Laura GÓMEZ, o.c. (1998), dedica la parte segunda de su libro en capítulos sucesivos al desarrollo de las actividades mencionadas, pp. 123 ss.

20 Se refiere a las cooperativas de consumo.

21 Texto recogido por Paul LAMBERT, o.c., pp. 176 y 177. 
chas revolucionarias, la superación del capitalismo en el tiempo, y el fomento de una economía participativa y democrática ${ }^{22}$.

Tiempo después escribe Gide en sus Principios de Economía Política que entre las cooperativas de producción, de presencia en Francia pero de éxito escaso, las de crédito y las de consumo, éstas «Las sociedades de consumo sobre todo tratan de absorber en ellas todas las demás formas y de realizar una especie de República Cooperativa en la que toda la dirección de la producción pasaría a manos de los consumidores, lo cual no sería ciertamente una revolución insignificante» ${ }^{23}$.

Posteriormente Ernest Poisson, que también formó parte de la Escuela de Nimes, publicó su obra más importante con el título «La república cooperativa», en 1920, para desarrollar en ella la primacía del consumidor y el programa de las tres etapas ${ }^{24}$. Dedica la obra al Profesor Charles Gide, el genio y apóstol de la república cooperativa25.

Con una cierta distancia, en 1952, André Hirshfeld, que había publicado un ensayo sobre Charles Gide en la Rivista de la Cooperazione ${ }^{26}$, consideraba que «El cooperativismo, como lo había soñado Gide y sus amigos, no será probablemente jamás realizado, pero sin embargo un sector cooperativo de día en día más importante, y más coherente, nos permite ya poner en práctica el ideal de fraternidad, de justicia y de libertad que nos transmitieron nuestros padres» 27.

\section{El cooperativismo en la política}

A pesar de la neutralidad política que predicaba la cooperativa de Rochdale y que quedó incorporada a los principios cooperativos desde los primeros momentos ${ }^{28}$, mantenida como principio recomendable pero no imprescindible en la reforma de los principios de la Alianza Cooperativa Internacional en 1937, y abandonada en la reforma posterior de 196629, el Partido Cooperativo Británico (The Co-operative

22 Puede verse Javier DIVAR GARTEIZ-AURRECOA, Filosofía de la cooperación económica (2012), p.160.

23 Véase Charles GIDE, Principes d'Économie Politique (1911), p. 481. Recoge también la frase en el Cours d'Économie Politique.

24 Puede verse Jorge OROZCO VILCHEZ, Antología: Doctrina cooperativa (1986), p. 179.

25 Ernest Poisson, The Co-operative Republic (1925).

26 Reproducido junto con el texto del prefacio de Albert Thomas para el libro de oro con el que se dio homenaje a Gide en 1927 con ocasión de sus ochenta años por INTERCOOP en 1967.

27 André HIRSCHFELD, Charles Gide (1967), p. 18.

28 Véase Paul LAMBERT, o.c., p. 57.

29 Sobre la evolución de los principios puede verse Alejandro MARTíNEZ CHARTERINA, «La cooperativa ...», o.c., pp. 213 ss. 
Party) fue constituido en 1917, y desde 1927 concurre a las elecciones conjuntamente con el Partido Laborista.

Se presenta como arma política del movimiento cooperativo, con más de 9.000 miembros, y trabaja para promover cooperativas y cualquier forma de organización mutual ${ }^{30}$. Tiene representación en las dos Cámaras del Parlamento, con 28 representantes elegidos, y más de 350 consejeros locales ${ }^{31}$.

El mismo año de nacimiento del Partido Cooperativo Británico, 1917, se fundó el México el Partido Nacional Cooperativista para fomentar el cooperativismo como forma de solución de los problemas económicos de las personas. Parece que aunque sus miembros llegaron a alcanzar algunos cargos importantes en los siguientes años, disensiones internas y algunas alianzas que no prosperaron llevaron a su pronta desaparición en $1923^{32}$.

Con todo, el caso de utilización de mayor relieve de la imagen cooperativa en la política de un Estado moderno corresponde, a juicio de Javier Divar, a la República Cooperativa de Guyana ${ }^{33}$.

Este país, colonia británica que alcanzó la independencia en 1966, adoptó el nombre de República Cooperativa en 1970 para poner de manifiesto su dirección hacia un socialismo cooperativista, todo ello de la mano del Presidente Linden Forbes Burnham, que a su muerte en 1985 dejó un país empobrecido y endeudado ${ }^{34}$.

\section{El modelo de la economía del bien común y la cooperativa ${ }^{35}$}

El planteamiento más actual en el que la cooperativa puede sentirse naturalmente involucrada es el que realiza Christian Felber en su obra La economía del bien común ${ }^{36}$.

30 Información en la página web del Partido Cooperativista Británico www.party.coop

31 Según noticia de Empresa y Trabajo.coop, periódico de COCETA, edición digital, n. ${ }^{\circ} 21,11$ de noviembre de 2010. Empresaytrabajo.coop/internacional/europa/el-partido-cooperativo-britanico-gana-terreno-en-el-panorama-politico-del-reino-unido/

32 Véase Memoria Política de México, en memoriapoliticademexico.org/Efemerides/8/07081917.html

33 Puede verse en Javier DIVAR GARTEIZAURRECOA, Reflexiones: Peter Cornelius Plockboy y la república cooperativa (2010), p. 150.

34 Ver Javier Divar, o.c. (2010), p. 151.

35 Recojo ideas que presenté en 2012 en la Ponencia marco de la Conferencia Desafíos de futuro para las cooperativas de consumo de la Confederación Española de Cooperativas de Consumidores y Usuarios (HISPACCOP).

36 Christian FELBER, La economía del bien común (2012). Pretende colocar la economía del bien común entre el sistema capitalista y el colectivista. Entre las distintas medidas que recoge está la implantación de un abanico salarial (concepto muy utilizado en las cooperativas 
La razón de ser de esta teoría es la contradicción que se produce entre una economía al servicio de las personas y una economía de mercado alejada de ese servicio: "....aunque los valores debieran ser la orientación esencial, las guías de nuestra vida, hoy en día en economía priman otros valores completamente diferentes a aquellos que son válidos en nuestras relaciones diarias con otras personas... la confianza, la sinceridad, el aprecio, el respeto, escuchar a los demás, la empatía, la cooperación, la ayuda mutua y la voluntad de compartir. La economía de libre mercado se basa en un sistema con normas que potencian la búsqueda de beneficios y la competencia. Estas pautas incentivan el egoísmo, la codicia, la avaricia, la envidia, la falta de consideración y de responsabilidad. Esta contradicción... nos divide en lo más profundo, como individuos y como sociedad ${ }^{37}$.

¿En qué consiste un buen resultado económico? En el momento presente el éxito económico se mide desde la perspectiva macroeconómica a través del Producto Interior Bruto, y desde la consideración microeconómica con el beneficio de la empresa.

Estos dos, PIB y beneficio, son indicadores monetarios, y el dinero no es capaz de medir en todo caso aquello que es importante para las personas a nivel global. Para las personas es importante conocer:

«—si un país está en guerra o en paz,

- si se trata de una dictadura o de una democracia,

— si el consumo de recursos medioambientales crece...

—si el reparto es justo...

—si las personas...disfrutan de suficiente tiempo libre,

—si las mujeres disfrutan de igualdad...

- si lo que crece en la sociedad es la confianza o el miedo» 38,

y desde la perspectiva de la empresa se trata de saber si

«-la empresa crea o destruye empleo,

— la calidad de los puestos de trabajo aumenta...

- los beneficios se reparten de manera justa,

- se trata y remunera igual a las mujeres y a los hombres,

- la empresa cuida o explota el medio ambiente,

- produce armas o alimentos ecológicos locales»39.

de trabajo) de 20 a 1 para evitar las alarmantes desigualdades existentes, que llegan a 325 a 1 en 2011 en los Estados Unidos (Juan Carlos Cubeiro, en la introducción a la obra citada de Felber, p. 11), que coincide con la denuncia de Paul Krugman, Acabad ya con esta crisis (2012), p. 84, que en 2006 los 25 gestores de fondos de Estados Unidos mejor pagados ganaros tres veces los sueldos de los 80.000 maestros de escuela de la ciudad de Nueva York.

37 Christian FELBER, O.C., p. 29.

38 Christian FELBER, o.c., p. 49.

39 Christian FELBER, o.c., p. 50. 
Aunque usamos en muchas ocasiones el PIB per cápita como indicador de bienestar no son pocas las críticas justificadas al mismo. En ese sentido se posiciona el informe de la Comisión de expertos sobre la medición de las actividades económicas y el progreso social, dirigido por Joseph Stiglitz, Amartya Sen y Jean-Paul Fitoussi, realizado por encargo del Presidente de Francia en $2008^{40}$. En el mismo sentido el uso cada vez más frecuente del Índice de Desarrollo humano que publica anualmente desde 1990 el Programa de Naciones Unidas para el Desarrollo (PNUD), que considera indicadores como la esperanza de vida al nacer, alfabetización y matriculación en la enseñanza, junto al PIB per cápita ${ }^{41}$.

Centrándonos en el ámbito microeconómico de la empresa ${ }^{42}$, el éxito económico de la misma deberá medirse en términos de bien común tales como la dignidad humana, la solidaridad, la sostenibilidad, la justicia social, la participación democrática y transparencia, todo ello con relación a los stakeholders, es decir, a los afectados por la actividad de la empresa, como son los proveedores, financiadores, empleados, propietarios, clientes, competidores, asociaciones locales, generaciones futuras, medio ambiente...

Es lo que recoge la matriz del bien común en la siguiente página. En ella se disponen en horizontal los valores que quieren considerarse como aportadores al bien común, y en vertical los grupos de relación con la empresa, procediéndose a atribuir puntos en el cruce de cada valor y grupo de relación. De este modo la matriz del bien común permite obtener puntos positivos, hasta 1.000 puntos, y negativos, hasta 900, que minoran los positivos ${ }^{43}$.

40 Publicado en España en 2013: Joseph STIGLITZ, Amartya SEN y Jean-Paul FITOUSSI, Medir nuestras vidas. Las limitaciones del PIB como indicador de progreso (2013).

41 Pueden verse todos los publicados en la página web siguiente: hdr.und.org/es/informes/mundial/idh2013.

42 Y dejando de lado las consideraciones macroeconómicas que exceden las pretensiones de este trabajo.

43 En la última versión de la Matriz del bien común, de 11 de abril de 2012 se han multiplicado los criterios negativos que pasan de cinco a dieciséis conceptos y, en términos de puntos, de 900 a 2.700. Se consideran nuevos criterios negativos los productos inhumanos (-200), la cooperación con empresas que lastiman la dignidad humana (-150), la patente defensiva (-100), el precio dumping (-200), el incumplimiento grave de especificaciones medioambientales $(-150)$, la obsolescencia programada $(-100)$, la reducción o desplazamiento de puestos de trabajo con beneficios $(-150)$, las filiales en paraísos fiscales (-200), los intereses al capital propio superiores al 10\% (-200), los impedimentos al comité de empresa (-150), y la no publicación de los flujos de filiales a lobbies (-200). Esta matriz está recogida en la dirección electrónica www.economia-delbien-comun.org . ISSN: 1134 - 993X, Núm. 47/2013, Bilbao, págs. 185-198 
El resultado total de puntos, positivos menos negativos, permite situar a cada empresa en uno de los cinco niveles, cada uno de ellos asociado a un color, siguientes ${ }^{44}$ :

\begin{tabular}{|l|l|}
\hline Nivel 1 & 0 a 200 puntos \\
\hline Nivel 2 & 201 a 400 puntos \\
\hline Nivel 3 & 401 a 600 puntos \\
\hline Nivel 4 & 601 a 800 puntos \\
\hline Nivel 5 & 801 a 1000 puntos \\
\hline
\end{tabular}

Matriz del bien común

\begin{tabular}{|c|c|c|c|c|c|}
\hline & Dignidad & Solidaridad & Sostenibilidad & Justicia social & Participación \\
\hline A. Proveedores & \multicolumn{5}{|c|}{ A1 Gestión ética de la oferta (90) } \\
\hline B. Financiadores & \multicolumn{5}{|c|}{ B1 Gestión ética de las finanzas (30) } \\
\hline C. Empleados & $\begin{array}{l}\text { C1 Calidad } \\
\text { puesto e } \\
\text { igualdad } \\
\mathbf{( 9 0 )}\end{array}$ & $\begin{array}{l}\text { C2 Reparto } \\
\text { justo del } \\
\text { trabajo } \\
\mathbf{( 5 0 )}\end{array}$ & $\begin{array}{l}\text { C3 Promoción } \\
\text { conducta } \\
\text { ecológica } \\
\mathbf{( 3 0 )}\end{array}$ & $\begin{array}{l}\text { C4 Reparto } \\
\text { justo de la } \\
\text { renta } \\
(\mathbf{6 0 )}\end{array}$ & $\begin{array}{l}\text { C5 Democracia } \\
\text { y transparencia } \\
(\mathbf{9 0 )}\end{array}$ \\
\hline D. Clientes & $\begin{array}{l}\text { D1 Venta ética } \\
\text { (50) }\end{array}$ & $\begin{array}{l}\text { D2 Solidaridad } \\
\text { con } \\
\text { copropietarios } \\
\text { (70) }\end{array}$ & $\begin{array}{l}\text { D3 } \\
\text { Concepción } \\
\text { ecológica de } \\
\text { productos y } \\
\text { servicios } \\
\mathbf{( 9 0 )}\end{array}$ & $\begin{array}{l}\text { D4 } \\
\text { Concepción } \\
\text { social de } \\
\text { productos y } \\
\text { servicios } \\
\text { (30) }\end{array}$ & $\begin{array}{l}\text { D5 Aumento } \\
\text { estándares } \\
\text { sociales y } \\
\text { ecológicos } \\
\text { (30) }\end{array}$ \\
\hline E. Ámbito social & $\begin{array}{l}\text { E1 Efecto } \\
\text { social del } \\
\text { producto - } \\
\text { servicio } \\
\mathbf{( 9 0 )}\end{array}$ & $\begin{array}{l}\text { E2 Aportación } \\
\text { al bien común } \\
\text { (40) }\end{array}$ & $\begin{array}{l}\text { E3 Reducción } \\
\text { de efectos } \\
\text { ecológicos } \\
\mathbf{( 7 0 )}\end{array}$ & $\begin{array}{l}\text { E4 } \\
\text { Minimización } \\
\text { reparto } \\
\text { ganancias a } \\
\text { externos } \\
\text { (60) }\end{array}$ & $\begin{array}{l}\text { E5 } \\
\text { Transparencia } \\
\text { social y } \\
\text { decisiones } \\
\text { participadas } \\
\mathbf{( 3 0 )}\end{array}$ \\
\hline Negativos & $\begin{array}{l}\text { Quebranta- } \\
\text { miento normas } \\
\text { laborales OIT } \\
\mathbf{( - 2 0 0 )}\end{array}$ & $\begin{array}{l}\text { Compra hostil } \\
(-\mathbf{2 0 0 )}\end{array}$ & $\begin{array}{l}\text { Gran impacto } \\
\text { medio- } \\
\text { ambiental } \\
\mathbf{( - 2 0 0 )}\end{array}$ & $\begin{array}{l}\text { Remuneración } \\
\text { desigual } \\
\text { mujeres- } \\
\text { hombres } \\
\text { (-200) }\end{array}$ & $\begin{array}{l}\text { No revelación } \\
\text { de participa- } \\
\text { ciones } \\
\mathbf{( - 1 0 0 )}\end{array}$ \\
\hline
\end{tabular}

Fuente: Christian FELBER, La economía del bien común (2012, a partir del cuadro «La matriz del bien común 4.0», pp. 58-59.

44 Christian Felber, o.c., p. 61, el nivel 1 asociado al color rojo, el 2 al naranja, el 3 al amarillo, el 4 al verde claro y el 5 al verde. 
Con la publicidad adecuada, es decir, informando a los consumidores, poniendo en los productos el color correspondiente junto al código de barras, la referencia a la matriz del bien común podrá orientar la confianza del público hacia el producto y la empresa, posibles reducciones fiscales por la aportación al bien común, prioridad en las compras públicas, y otras respuestas favorables.

El año 2010 elaboraron el balance del bien común setenta empresas, de las cuatrocientas empresas y setenta organizaciones que apoyaban el modelo. En la actualidad el número de simpatizantes ha aumentado hasta 1.264 empresas y 157 asociaciones, estando los grupos de apoyo más notables en Austria, Alemania, Italia, Suiza, España y América latina ${ }^{45}$.

Considerando los valores que promociona la economía del bien común así como los grupos de relación en los que se miden resultados, no debe extrañar la presencia de las cooperativas en la mayor parte de los ejemplos de empresa de bien común que, revisando diversas partes del mundo, realiza Christian Felber ${ }^{46}$, empezando por la Corporación Mondragón.

Y es que el enlace de la cooperativa, poseedora de una identidad de valores y principios de comportamiento en los que la propiedad conjunta y la gestión democrática forman parte esencial de la misma, con la economía del bien común es absoluto. Y en la medida en que la economía del bien común pretende abordar no solamente los aspectos microeconómicos sino también los globales de una sociedad se vuelve a poner de manifiesto la voluntad del cooperativismo por caminar hacia un mundo más justo y solidario, como en tantas ocasiones se ha puesto de manifiesto.

\section{Conclusión}

La cooperativa es una empresa singular de autoayuda. Pero no se agota en si misma sino que se prolonga en un movimiento cooperativo a través del cual esa singularidad se extiende.

Por otra parte los valores y principios de las cooperativas, a los que ajustan su funcionamiento, les conducen a una constante preocupación y atención por su entorno, tanto por las personas como por el mismo medio ambiente en el que están emplazadas y desarrollan su actividad.

45 Ver en Christian FELBER, o.c., p. 13, y www.economia-del-bien-comun.org

46 En el capítulo séptimo de la obra citada, ps. 189 ss. 
Esto lo tiene en cuenta la Alianza Cooperativa Internacional tanto en la custodia e interpretación de la identidad cooperativa, como en las llamadas aspiraciones de conquista del cooperativismo que le vinculan con pretensiones que trascienden el ámbito de la cooperativa y su entorno para buscar una transformación social hacia un mundo mejor y más justo.

Y en el momento actual en el que se promociona el modelo de la economía del bien común, la cooperativa encuentra un marco de referencia en el que puede tener, desde su propia naturaleza, un perfecto asentamiento, y constituir un tipo de empresa perfectamente acomodada en el citado modelo.

\section{Bibliografía}

ACl: Declaración de la Alianza Cooperativa Internacional sobre la identidad cooperativa, Consejo Superior de Cooperativas de Euskadi, Vitoria-Gasteiz, 1996.

ACl: Estatuto (2009), www.ica.coop/sites/default/files/attachments/2009-09ICA-Statutes-Spanish.pdf.

DAVIDOVIC, Georges: Hacia un mundo cooperativo, CENEC, Zaragoza, 1976.

DIVAR GARTEIZ-AURRECOA, Javier: "Filosofía de la cooperación económica», en Boletín de la asociación Internacional de Derecho Cooperativo, n. ${ }^{\circ} 46$, Universidad de Deusto, Bilbao, 2012, pp. 147-160.

DIVAR GARTEIZ-AURRECOA, Javier: «Reflexiones: Peter Cornelius Plockboy y la república cooperativa», en REVESCO, n. ${ }^{\circ} 102$, segundo cuatrimestre 2010, ps. 145-153 (www.ucm.es/info/revesco).

DIGBY, Margaret: El movimiento cooperativo mundial, Editorial Pax, México, 1965.

FELBER, Christian: La economía del bien común, Deusto - Grupo Planeta, Barcelona, 2012.

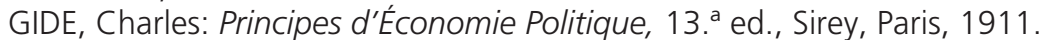

GÓMEZ, Laura: La Alianza Cooperativa Internacional y su desarrollo como institución y en especial como instrumento transformador de la sociedad, Consejo Superior de Cooperativas de Euskadi, Vitoria-Gasteiz, 1998.

KAPLAN DE DRIMER, Alicia y DRIMER, Bernardo: Las cooperativas: fundamentos, historia, doctrina, 3. ${ }^{\text {a }}$ ed., INTERCOOP, Buenos Aires, 1981.

KRUGMAN, Paul: Acabad ya con esta crisis, Crítica, Barcelona, 2012.

LAIDLAW, A.F.: "Las cooperativas en el año 2000», en Tribuna Cooperativa, n. $.^{\circ} 4-45$, CENEC, Zaragoza, 1982, ps. 11 a 152.

LAMBERT, Paul: La doctrina cooperativa, 3. ${ }^{a}$ ed., INTERCOOP, Buenos Aires, 1970.

Laws and objets of the Rochdale Society of Equitable Pioneers: enrolled according to the Acts, $10^{\text {th }}$, George IV, and $4^{\text {th }}$ and $5^{\text {th }}$, William IV. Printed by Jesse Hall, Rochdale, 1844. 
MARTÍNEZ CHARTERINA, Alejandro: "La cooperativa como empresa y los principios cooperativos tras el congreso centenario de la A.C.I. de Manchester», en Evolución del escenario económico, Universidad de Deusto, Bilbao, 1996, ps. 207-226.

MARTÍNEZ CHARTERINA, Alejandro: "Evolución del cooperativismo de consumo», en Boletín de la Asociación Internacional de Derecho Cooperativo, n. ${ }^{\circ} 45$, Universidad de Deusto, Bilbao, 2011, ps. 133-160.

MLADENATZ, Gromoslav: Historia de las doctrinas cooperativas, INTERCOOP, Buenos Aires, 1969.

OROZCO VILCHEZ, Jorge: Antología: Doctrina cooperativa, EUNED, San José de Costa Rica, 1986.

POISSON, Ernest: The Co-operative Republic, The Co-operative Union Limited, Manchester, 1925.

SAPELLI, Giulio (A cura di): II movimiento cooperativo in Italia: Storia e problemi, Einaudi, Torino, 1981.

STIGLITZ, Joseph E.; SEN, Amartya; y Fitoussi, Jean-Paul: Medir nuestras vidas. Las limitaciones del PIB como indicador de progreso, RBA, Barcelona, 2013.

WATKINS, W.P.: L'Alliance Cooperative International 1895-1970, A.C.I., Londres, 1971.

WATKINS, W.P.: El movimiento cooperativo internacional, INTERCOOP, Buenos Aires, 1977. 